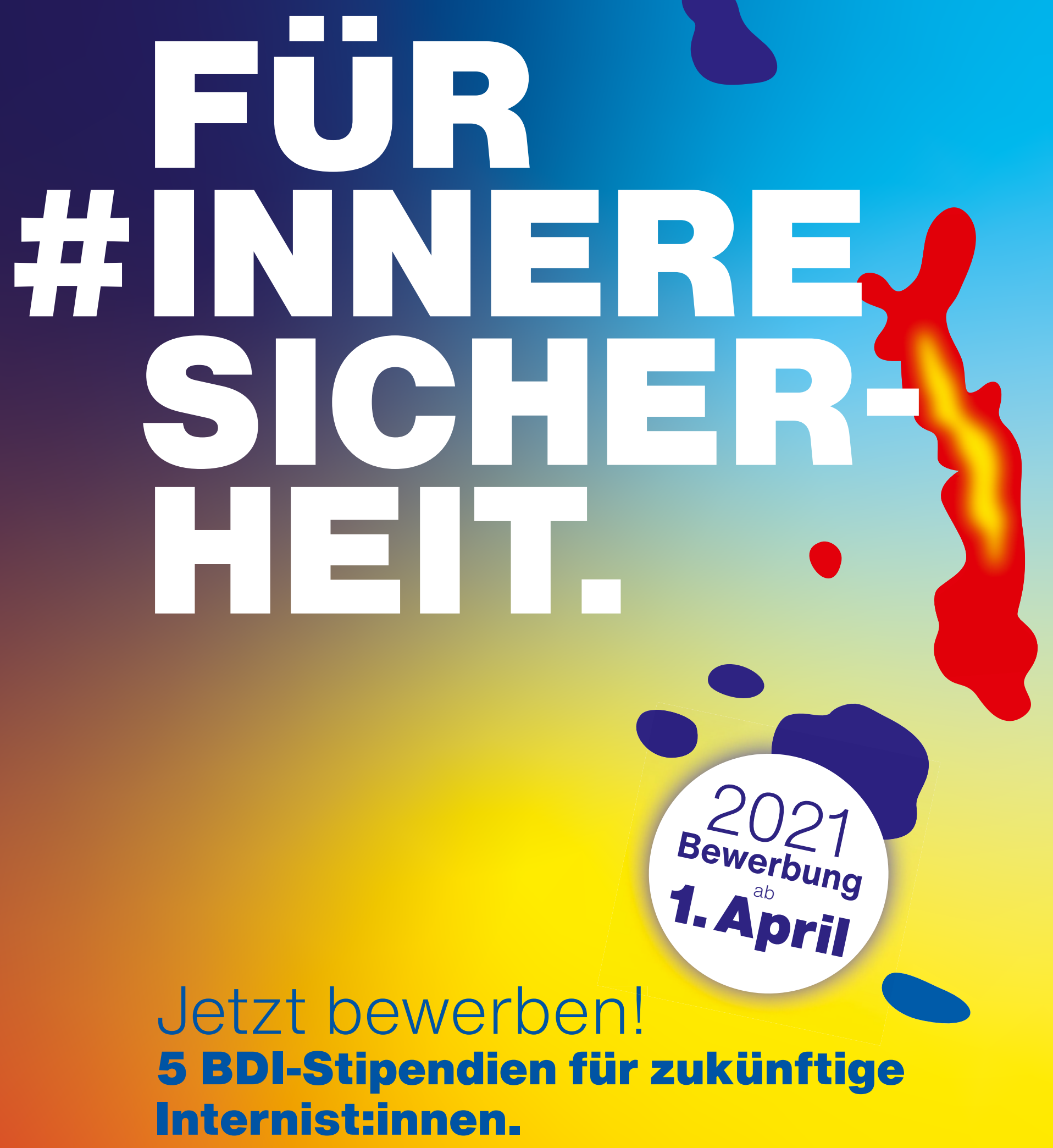

Bewerben können sich alle immatrikulierten Studierenden an deutschen Universitäten der Fachrichtung „Humanmedizin“. Die fünf zu vergebenden BDI-Stipendien unterstützen Bewerber:innen, die ihre berufliche Zukunft in der Inneren Medizin sehen und sich gerne im Verband engagieren.

Voraussetzung für die Förderung ist der erfolgreiche Abschluss des Physikums.

www.bdi.de/nachwuchs/studium/stipendium

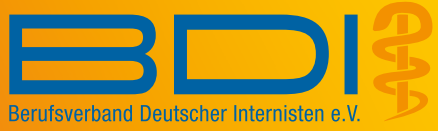


Internist 2021 · 62:526-532

https://doi.org/10.1007/s00108-021-01033-w

(c) Springer Medizin Verlag GmbH, ein Teil von Springer Nature 2021

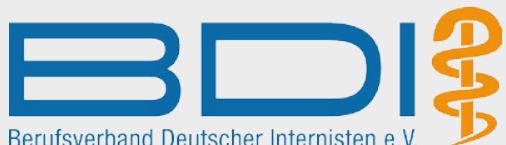

Berufsverband Deutscher Internisten e.V.
Redaktion

Christine Neumann-Grutzeck, Wiesbaden (v.i.S.d.P.)

Korrespondenzadresse

Berufsverband Deutscher Internisten e.V.

Schöne Aussicht 5

65193 Wiesbaden

Tel.: 0611 18133-0

Fax: 0611 18133-50

info@bdi.de|www.bdi.de
Folgen Sie uns auf

in @bdi-e-v

@ @BDI_eV

(f) @lhr.BDI

(요 @bdi_ev

\begin{tabular}{|ll}
\hline \multicolumn{2}{|l|}{ Inhalt } \\
\hline $\mathbf{5 2 7}$ & Keine Zeit für Kompetenzen \\
\hline $\mathbf{5 2 8}$ & Interview:,Wir brauchen eine neue Weiterbildungskultur" \\
\hline $\mathbf{5 2 9}$ & Ärztliche Weiterbildung darf nicht zur Ware verkommen! \\
\hline $\mathbf{5 2 9}$ & Zweite Facharztprüfung: Jede Kammer entscheidet selbst \\
\hline $\mathbf{5 3 0}$ & Der Mindeststandard als Objekt naiver Sehnsucht \\
\hline $\mathbf{5 3 1}$ & $\begin{array}{l}\text { Anstellung in Vertragsarztpraxis ohne Facharztqualifizierung nicht } \\
\text { möglich }\end{array}$ \\
\hline
\end{tabular}

\section{Thema: Weiterbildung}

\section{Keine Zeit für Kompetenzen}

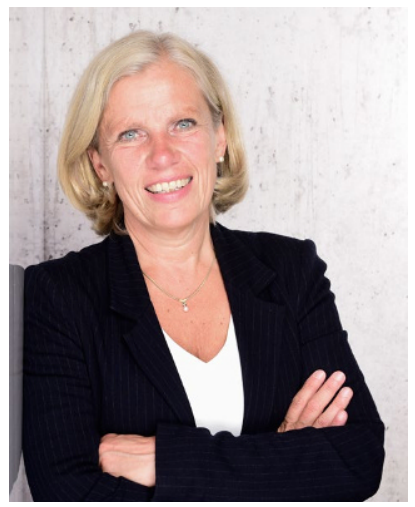

Liebe Kollegen und Kollegen,

die ärztliche Weiterbildung hat durch die Corona-Pandemie vielerorts gelitten. Unter Pandemiebedingungen gestaltet es sich deutlich schwieriger, Facharztkompetenzen zu erwerben. Außerplanmäßige Einsätze auf COVID-19-Stationen verzögern die Weiterbildung ebenso wie ausbleibende Rotatio- den Weg in die Weiterbildungsordnung gefunden. Dabei ist es für die Ärztekammern deutlich schwieriger, erworbene Kompetenzen anstelle abgeleisteter Zeiten zu überprüfen. Die fachlich empfohlenen Weiterbildungspläne sollen dabei helfen.

Für Ärzt:innen in Weiterbildung besteht mit der kompetenzbasierten Weiterbildung ein nicht unerheblicher Dokumentationsaufwand. Das E-Logbuch soll die Dokumentation erleichtern sowie die Planung der Weiterbildung und das jährliche Weiterbildungsgespräch unterstützen, indem Fortschritte und Defizite sichtbar werden können. Ein anzustrebendes Ziel wäre auch, das E-Logbuch für die systematische Evaluation der Weiterbildung zu nutzen. Dies ist jedoch an die freiwillige Freigabe der Daten durch die Weiterzubildenden gebunden und aus datenschutzrechtlichen Gründen aktuell noch nicht flächendeckend umsetzbar.

Letztendlich zählt jedoch nur eins: Ärzt:innen in Weiterbildung müssen die von ihnen geforderten Kompetenzen auch vermittelt bekommen. Gute Weiterbildung benötigt deshalb Verlässlichkeit und Planbarkeit - das gilt für Weiterzubildende wie für Weiterbilder:innen gleichermaßen - aber auch ausreichend Zeit der Weiterbilder:innen, angehende Fachärzt:innen beim Kompetenzerwerb zu unterstützen. Der BDI setzt sich deshalb schon lange dafür ein, eine:n Weiterbildungsoberärzt:in mindestens teilweise für diese Funktion freizustellen.
Für viele Kliniken wird gute Weiterbildung auch immer mehr zu einem Standortvorteil im Ringen um kompetente Mitarbeiter:innen. In Zeiten des Ärzt:innenmangels schauen die jungen Kolleg:innen sehr genau, was ihnen die Abteilungen bieten können. Konkurrenz droht aus dem ambulanten Versorgungsbereich, denn die neue WBO ermöglicht deutlich mehr Optionen, Teile der Weiterbildung ambulant abzuleisten. Das betrifft alle Facharztweiterbildungen. Dafür ist es notwendig, die richtigen Rahmenbedingungen zu schaffen, z. B. mit Hilfe einer entsprechenden Finanzierung, die aktuell nur für die Allgemeinmedizin umfassend gesichert ist. Hier ist ein Umdenken erforderlich, da immer mehr Weiterbildungsinhalte stationär gar nicht mehr vermittelt und damit erworben werden können - eine Entwicklung, die positiv und besorgniserregend zugleich ist.

Zur Sicherstellung einer guten Patientenversorgung ist eine hochwertige Weiterbildung sowohl stationär als auch ambulant unentbehrlich. Deshalb müssen wir auf allen Ebenen - personell wie finanziell - in unseren fachärztlichen Nachwuchs investieren.

Ihre

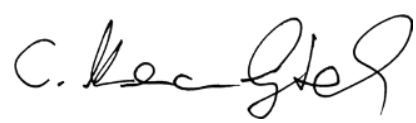

Christine Neumann-Grutzeck Präsidentin des BDI 


\section{Interview}

\section{„Wir brauchen eine neue Weiterbildungskultur"}

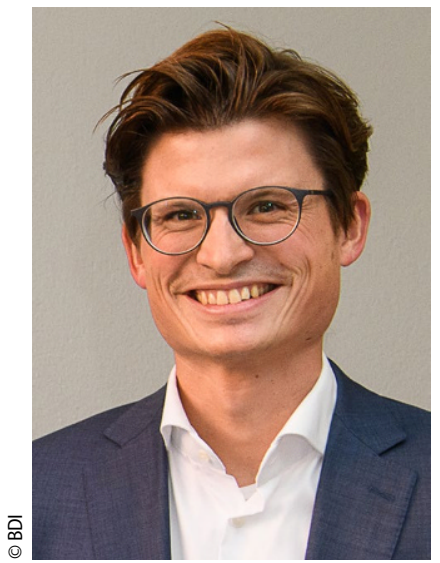

In Schleswig-Holstein ist es möglich, in sieben Jahren sowohl den Facharzt für Innere Medizin als auch einen Schwerpunktfacharzt zu erwerben. Der BDI sprach mit Kammerpräsident Prof. Henrik Herrmann darüber, was diese Doppelqualifikation jungen Ärzten bringt. Und warum nicht alle Kammern die verkürzte zweite Weiterbildung anbieten.

Das Interview führte der 2. BDIVizepräsident Dr. med. Kevin Schulte mit dem Präsidenten der Ärztekammer Schleswig-Hollstein Prof. Dr. med. Henrik Herrmann.

Dr. Kevin Schulte: Herr Professor Herrmann, Sie sind selbst Internist und blicken auf eine langjährige Tätigkeit zurück. Was ist aus Ihrer Sicht das Besondere an der Inneren Medizin?

Professor Henrik Herrmann: Ich habe 1984 mit der Inneren Medizin angefangen. Mich hat dabei die Breite fasziniert: sowohl der wissenschaftliche als auch der menschliche Teil der Medizin, also die sozialen, kommunikativen Aspekte, sind in der Inneren sehr ausgeprägt. Ich habe immer den Anspruch gehabt, am Ende meiner Weiterbildungszeit sagen zu können: Ich kann jeden internistischen Patienten die ersten 24 Stunden alleine betreuen. Des-

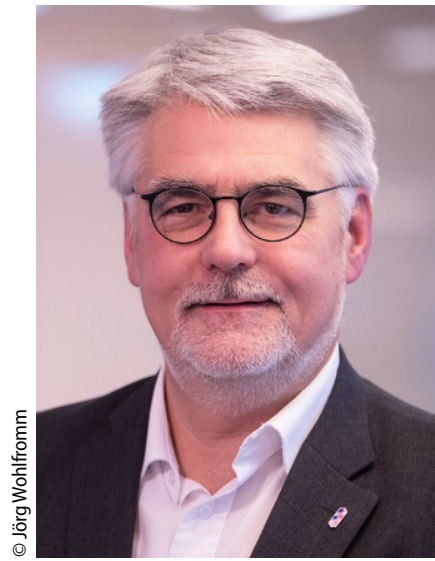

halb bin ich auch nach wie vor Allgemeiner Internist, ich habe das immer als Vorteil empfunden. Gerade auch, als ich dann als Chefarzt an ein Krankenhaus der Grund- und Regelversorgung gewechselt bin.

Das heißt, Sie würden einer jungen Kollegin bzw. einem jungen Kollegen raten, zunächst den Facharzt für Innere Medizin zu machen, bevor man sich für einen Schwerpunkt entscheidet?

Primär ja. Es sei denn, ich weiß von mir, dass ich mein Leben lang etwa nur kardiologisch spezialisiert tätig werden will. Ich glaube aber, dass viele Kolleginnen und Kollegen, die mit der Allgemeinen Inneren Medizin beginnen, sich anfangs noch gar nicht so genau festlegen können.

Andererseits ist so ein $\mathrm{Be}$ rufsweg, wie ich ihn gegangen bin, heute nicht mehr realisierbar. Diesen Anspruch, den ich in den 80er Jahren an mich selbst hatte, kann man durch den zunehmenden Fortschritt in der Inneren Medizin heute nicht mehr abbilden. Es ist gar nicht mehr möglich, alle Teilgebiete zu überblicken. Deshalb braucht man für seinen Karriereweg eine Spezialisierung, aber der Grundstock bleibt die breite Innere Medizin.
Kürzlich haben Sie gesagt, der Facharzt für Innere Medizin habe Konjunktur...

Ja, 2019 haben insgesamt 2100 Kolleginnen und Kollegen den Facharzt für Allgemeine Innere Medizin gemacht - damit liegt dieser bundesweit an erster Stelle. Mit gut 400 Facharztprüfungen weniger folgt auf Rang zwei die Allgemeinmedizin und auf Rang drei die Anästhesie, die sogar über 700 Facharztprüfungen weniger als die Allgemeine Innere Medizin zählt. Dabei verzeichnet die Allgemeine Innere Medizin schon seit mehreren Jahren die höchsten Steigerungsraten. Interessant ist auch der Anteil der Ärztinnen an den Prüflingen: In der Allgemeinen Inneren Medizin machten sie 2019 mit $55 \%$ über die Hälfte aus, bei den Spezialisierung in der Inneren Medizin lediglich $44 \%$.

Es heißt ja immer, zu wenige junge Mediziner interessieren sich für die hausärztliche Versorgung. Wenn 2100 Ärztinnen und Ärzte pro Jahr die Kompetenz erlangen, im hausärztlichen Bereich tätig zu werden, dann ist das doch ein sehr positives Signal.

$\mathrm{Ja}$, das ist ein gutes Zeichen. Dabei sehen wir auch in den Spezialitäten, dass die Prüfungen konstant sind, also nicht zurückgehen. 2019 wurden hier 1352 Facharztprüfungen abgelegt. Die Arztzahlen steigen insgesamt.

In Schleswig-Holstein haben Sie sich dafür stark gemacht, dass es möglich ist in sieben Jahren sowohl den Facharzt für Innere Medizin als auch einen Schwerpunktfacharzt zu erwerben. Wie kommt es, dass das nicht in allen Kammergebieten möglich ist?

Wir sind in der Weiterbildung immer noch an Zeiten gebunden. Daher kommt es darauf an, welche Basis ich bei diesen Zeiten zugrunde lege. Die meisten Kammern orientieren sich an der deutschen Weiterbildungsordnung. Dazu muss man wissen, dass man bei Erwerb eines zusätzlichen Facharztes mindestens die Hälfte der Mindestdauer der Weiterbildung in diesem Fach absolvieren muss. Nimmt man die deutsche Weiterbildungszeit, dann sind das für den Allgemeinen Internisten fünf Jahre und zum Beispiel für den Facharzt Innere Medizin und $\mathrm{Ne}$ phrologie sechs Jahre. Das heißt, macht man erst den Allgemeinen Internisten, müsste man drei Jahre Nephrologie draufsetzen, auch wenn ich in meiner allgemeinen Weiterbildung vielleicht schon nephrologische Inhalte abgedeckt habe.

In Schleswig-Holstein halten wir uns an die europäische Richtlinie über die Anerkennung von Berufsqualifikationen (2005/36/EG). Diese weist aus, dass sich beim Erwerb einer weiteren Facharztbezeichnung die festgelegte Weiterbildungszeit verkürzen kann, wenn abzuleistende Weiterbildungszeiten bereits im Rahmen einer anderen fachärztlichen Weiterbildungsbezeichnung absolviert worden sind, wobei die mögliche Anrechnung auch die Hälfte der jeweiligen Facharztweiterbildung entspricht. Diese Zeiten sind aber im europäischen Kontext geringer: beim Nephrologen sind es vier Jahre, beim Angiologen sogar nur drei Jahre. Das heißt, man würde die Allgemeine Innere Medizin mit fünf Jahren machen und für den Nephrologen dann eben noch einmal zwei Jahre oben drauf, also insgesamt sieben Jahre.

Nun hat ja die neue Musterweiterbildung den Kompetenzbegriff in den Mittelpunkt gestellt. Wäre es dann nicht sinnvoll, dass deutschlandweit in einer ersten Facharztweiterbildung erworbene Erkenntnisse auch auf die zweite Facharztweiterbildung anerkannt werden können?

Das ist vollkommen richtig. Durch die Kompetenzbasierung ist hinterlegt, dass ich Inhalte mitnehmen kann. Diese werden auch im eLogbuch dokumentiert, können also nicht verloren gehen. Warum Landes- 
ärztekammern diesen Weg, den wir in Schleswig-Holstein - übrigens mit Zustimmung unserer Aufsichtsbehörde - gegangen sind, nicht einschlagen, das müsste in den einzelnen Kammern durchaus diskutiert werden. Durch Bezugnahme auf die europäische Richtlinie kann nämlich die Weiterbildungszeit reduziert werden, natürlich ohne die erworbene Kompetenz zu reduzieren.

Neben Ihrer Rolle als schleswigholsteinischer Ärztekammerpräsident sind Sie Co-Vorsitzender der Ständigen Konferenz "Ärztliche Weiterbildung" der Bundesärztekammer. Spielt dieses Thema auch dort eine Rolle?

Das wurde natürlich in den vergangenen Jahren mehrfach diskutiert. Wie so oft, ist es ein Kompromiss, weil sich noch nicht jede Kammer dazu durchringen konnte. Wir müssen aber auch mit der kompetenzbasierten Weiterbildung erst noch Erfahrungen sammeln. Wir merken, dass die Befugniserteilung

\section{Zweite Facharztprüfung: Jede Kammer entscheidet selbst}

Die Abkürzung der zweiten Facharztweiterbildung durch Anerkennung von Leistungen der ersten Facharztweiterbildung ist in den jeweiligen Landesweiterbildungsordnungen geregelt. Danach werden Leistungen aus der ersten Weiterbildung grundsätzlich anerkannt. Allerdings darf "die noch abzuleistende Weiterbildungszeit höchstens um die Hälfte der Mindestdauer der jeweiligen Facharztweiterbildung reduziert werden", so der Wortlaut der Weiterbildungsordnungen der meisten Kammerbezirke. Danach darf also die zweite Weiterbildung auf die Hälfte der Dauer gekürzt werden.

In vier Kammerbezirken finden sich jedoch abweichende Regelungen, nach denen die noch abzuleistende Weiterbildungszeit höchstens um die Hälfte der Mindestdauer der Facharztweiterbildung nach der Europäischen Weiterbildungsrichtlinie (RL 2005/36/EG) reduziert werden darf. Dies ist in Hessen, in beiden Kammerbezirken in NordrheinWestfalen und in Schleswig-Holstein der Fall.

Die Dauer der Weiterbildungen nach der EU-Richtlinie ist meist deutlich kürzer als die nach nationalem Recht. Beispielsweise ist für die Weiterbildung zum Nephrologen in Deutschland die Weiterbildungsdauer von sechs Jahren vorgesehen, nach der EU-Richtlinie dauert die Weiterbildung für „Nierenkrankheiten“ jedoch lediglich vier Jahre. So kann es dazu kommen, dass in den genannten vier Ärztekammerbezirken die zweite Facharztweiterbildung in nur zwei Jahren durchlaufen werden kann, während Ärztinnen und Ärzte in den übrigen Kammerregionen hierfür drei Jahre einplanen müssen. (Monika Roski, Rechtsanwältin, Assistenz der Geschäftsführung und Justitiarin des BDI) dungskultur! (Mitarbeit Rebekka Höhl, Teamleiterin Gesundheitspolitik, Ressort Gesundheitspolitik \& Wirtschaft, Ärzte Zeitung)

\section{Professor Henrik Herrmann}

Präsident der Ärztekammer

Schleswig-Holstein (seit 2018)

Mitglied der Ständigen

Konferenz Weiterbildung der

Bundesärztekammer (seit 2001)
Ausbildung: 1977-1984

Studium der Medizin in Kiel; 1984 -1991 Weiterbildung zum Facharzt für Innere Medizin, Westküstenklinikum Heide; Zusatzweiterbildungen: Geriatrie, Palliativmedizin, Diabetologie, Intensivmedizin, Notfallmedizin, Ärztliches Qualitätsmanagement

\section{Ärztliche Weiterbildung darf nicht zur Ware verkommen!}

Mittelpunkt ärztlicher Weiterbildung sollte der Patient sein. Bewertungsplattformen für Weiterbildungsstätten sind da eher hinderlich.

Schon der Deutsche Ärztetag hat beschlossen, dass Weiterbildung nicht eine Aneinanderreihung von Zahlen ist. In der neuen Weiterbildung werden kognitive und Handlungskompetenzen vermittelt und durch eine Prüfung verifiziert. Nur diese Kompetenzen ermöglichen ein qualifiziertes ärztliches Handeln und Patientensicherheit. Natürlich gibt es auch während der Weiterbildungszeit unterschiedliche, auch wirtschaftliche, Interessen. Diese müssen analysiert, eingeordnet und ausgeglichen werden, damit Weiterbildung nicht zur beliebigen Ware verkommt.

Für die/den Weiterzubilden$\mathrm{de} / \mathrm{n}$ ist es heute zum Glück etwas einfacher als auf dem Wohnungsmarkt. Fachrichtung und Weiterbilder können frei gewählt werden, die Vergütung ist tariflich festgelegt. In Hessen ist die tarifliche Vergütung sogar Teil der Weiterbildungsordnung. Doch es geht nicht nur um Weiterbildungsstätte und -fach. Die Weiterbildung selbst und damit die Versorgung von Patienten sind ebenfalls keine Ware. Sie beruhen zunächst auf der ärztlichen Kooperation zwischen Weiterbilder und Weiterzubildendem in
Klinik und Praxis. Dieses grundsätzliche Vertrauensverhältnis ist für die Vermittlung der Kompetenzen ebenso erforderlich, wie für die Versorgung der anvertrauten Patienten. Bewertungen, wie heute im Internet üblich, und daraus abgeleitete Rankings lassen die Weiterbildung jedoch genau als solches erscheinen: eine beliebig tauschbare Ware.

\section{Nicht sicher vor Fake News}

Der Umfang einer möglichen Stichprobe ist meist zu klein für eine valide Aussage, das Interesse der Plattform und der Bewerter wird nicht deutlich. Politisch gewollt ist womöglich eine nicht kontrollierbare Prangerfunktion für Weiterbilder und ihre Träger. Fehlbewertungen und Fake News sind nicht ausgeschlossen, Korrekturen faktisch unmöglich.

Selbstverständlich unterliegt die Weiterbildung - im Patienteninteresse - einer Qualitätssicherung. Als Voraussetzung für die Weiterbildung müssen die Befugten persönlich und fachlich geeignet sein und über eine einschlägige Erfahrung verfügen. Inhaltliche Dissonanzen zur Weiterbildung können über die Ärztekammer ausgeräumt werden. Mit dem neuen E-Logbuch lässt sich, nur auf Wunsch der Weiterzubildenden, dabei rasch ein Überblick über den 
Stand der Weiterbildung gewinnen. Eine unzureichende Weiterbildung wird durch individuelle Hinweise der Kammer und ggf. durch Entzug der Befugnis oder eine Verlängerung der Weiterbildungszeit sanktioniert.

Gemeinsames politisches Ziel der Ärzteschaft ist eine qualifizierte ärztliche Weiterbildung, die nicht von wirtschaftlichen Interessen und fremden Einflüssen dominiert wird. DRG-System und EBM-Strukturen sind kontraproduktiv für die Weiterbildung. Förderungen einzelner Weiterbildungsbereiche durch die Politik alleine ergeben Fehlanreize. Der Patient muss auch in der Weiterbildung wie- der in den Mittelpunkt gerückt werden.

Warum Ärzte Weiterbilden? - Die Vergütung von Kollegen in Weiterbildung ist zwar niedriger als die eines Facharztes. Eine Vielzahl von ärztlichen Leistungen ist aber auch ohne Facharztstatus erbringbar, damit ergeben sich für Kliniken, MVZ und Praxen, die weiterbilden, durchaus Kostenvorteile.

\section{Dr. med. Wolf-Andreas Fach} Vorsitzender BDI-LV Hessen

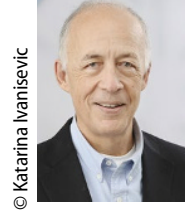

Pläne über die Dienst- und Einsatzzeiten der jungen Kolleginnen und Kollegen gibt, aber kein passendes Pendant die Weiterbildung betreffend. Die Folge: Der Mindeststandard ist zum Objekt tiefer Sehnsucht geworden. Ein Assistent, der in der Regelzeit tatsächlich alle seine (Mindest-)Untersuchungen zusammen hat, ist der König auf jeder Party und sein Geheimnis, wie er das geschafft hat, in sozialen Medien viel wert.

Im Jungen Forum des BDI, sowie in den jungen Kammern und Arbeitskreisen der Landesärztekammern werden in der Regel andere Drehbücher geschrieben: „Guten Morgen Herr Kollege, viel los heute, hm?", „Ja.“. Folge zu Ende.

\section{Der Mindeststandard als Objekt naiver Sehnsucht}

\begin{abstract}
Mit der Weiterbildungsnovelle ändert sich für die kommende Ärztegeneration die wichtigste Grundlage ihrer Ausbildung. Wo stehen wir aktuell? Wer sind wir jungen Ärzte? Und was wollen wir?
\end{abstract}

„Guten Morgen Herr Kollege, dann lassen sie uns mal loslegen!" Der Oberarzt winkt mit einer herzlichen und routinierten Geste den ersten Patienten rein. „So Herr Miller, machen sie mal das T-Shirt hoch, genau." Jovial legt der Oberarzt die rechte Hand auf die Schulter des Assistenzarztes, während er mit der linken auf dem Sono-Bildschirm die relevanten Strukturen zeigt. „Genau, wie besprochen, ein bisschen nach links kippen, genau." Diese Szene, Sie haben es vermutlich erraten, stammt nicht aus einem Deutschen Krankenhaus, sondern aus einer bekannten US-amerikanischen Klinikserie. Sie trifft aber in etwa die Idee von Weiterbildung in den Köpfen junger Ärztinnen und Ärzte bzw. von denen, die es bald sein werden.

\section{Der Arzt ist auch nur ein Mensch}

Diese wenig spektakuläre Szene drückt eine Sehnsucht aus, die so trivial wie bedeutend zugleich ist: Ärzte, unabhängig ihrer Hierarchieebene, als Team zu begreifen, Raum und Zeit für Teaching unter echten Bedingungen und die Idee, den Nachwuchs als echte internistische Arbeitshilfe zu begreifen und nicht nur als Aufseher einer anonymen ArztbriefManufaktur. Es ist ein tiefes Bedürfnis eines jeden Menschen, so auch der Weiterzubildenden, Anteil am Fortkommen der Gruppe zu haben. Je relevanter der eigene Anteil, desto besser. Studien, die einen Mangel und die daraus resultierenden beruflichen Gratifikationskrisen junger Ärzte zeigen, wurden bereits zu Genüge in „BDI aktuell“ und vielen weiteren Publikationen zitiert.

Die Umwälzungen des Gesundheitssektors in den letzten Jahrzehnten haben mit ihrer zunehmenden Arbeits- und Dokumentationsverdichtung dazu geführt, dass es zwar detaillierte
Weiterbildung während der Pandemie nicht ausreichend fortsetzen zu können.

\section{Generation Y(es - we can!)}

Wer sind diese Burn-out gefährdeten jungen Leute eigentlich und wieso schreiben sie nicht einfach ihre Arztbriefe, bis man das Zeugnis unterschreibt? Im Gegensatz zu vielen Vorurteilen zeichnet sich die jetzige Generation der Ärztinnen und Ärzte in Weiterbildung neben ihrem Hang zum Overcomittment (Überengagement, $60 \%$ ) vor allem durch eine tiefe Identifikation mit ihrer Tätigkeit aus. Über 60 \% der jungen Arbeitnehmer der Generation Y geben als Hauptgrund für ihre Tätigkeit die Verbesserung der Gesellschaft an. $94 \%$ möchten ihre Fähigkeiten dazu einsetzen, einen Mehrwert für die Gesellschaft zu schaffen. Klingt naiv? Ist es vielleicht auch. Es ist vor allem aber eine Chance. Eine Chance, die wir nicht ungenutzt lassen sollten.

Eine Diskussionsrunde des Bündnis Junge Ärzte beim SpiFa-Fachärztetag brachte es auf den Punkt: Das alte Modell des Einzelkämpfers in der Praxis läuft aus. Die junge Generation wünscht sich kooperative Strukturen und setzt auf andere Werte: Work-Life-Balance und Flexibilität, Vereinbarkeit von Familie und Beruf, Vernetzung, Transparenz und weniger Bürokratie. Hierbei stellt Selbstständigkeit keinen Widerspruch dar, viel mehr könne man durch neue flexible und vernetzte Strukturen eine Art Selbstständigen-Synzytium schaffen. Dass hierfür die Digitalisierung vorangetrieben werden müsse sei selbstverständlich.

Als kritischer Beobachter mag man sich vielleicht zu dem Gedanken hinreißen lassen, dass auch das naiv sei, da es sicherlich in einem solchen Konstrukt zu Interessenskonflikten der Parteien kommen müsse. Wer so denkt, vergisst, mit welcher Generation und welchen Motiven er es zu tun hat. 


\section{Novelle als Gamechanger}

Die neue Weiterbildungsordnung rückt die Kompetenzen in den Vordergrund. In einem mehrstufigen Prozess können sich der Weiterzubildende sowie der Weiterbilder darüber verständigen, ob bestimmte Kompetenzen vorliegen oder nicht. Dieser Austausch soll regelmäßig erfolgen und nicht erst am Ende der gesamten Weiterbildung.

Dies bringt gleich mehrere Vorteile mit sich, die das Zeug dazu haben, tiefgreifende Veränderungen in Gang zu bringen: Zum ersten Mal findet echte, institutionalisierte Prozesskontrolle (im Gegensatz zur bisherigen Ergebniskontrolle) statt. Charmanter Weise erfolgt die Kontrolle im Team (Weiterbilder/Assistent) und nicht primär durch die Kammer (außer es wird ex- plizit gewünscht). So kann wieder zusammenwachsen, was in der Medizin Tradition hat: Lehrer und Lernender. Falls es doch zu Problemen kommen sollte, können die Ärztekammern anderseits nun sehr viel leichter und schneller anhand von echten $\mathrm{Da}$ ten gemeinsam mit den Beteiligten Lösungen finden.

Durch diesen ritualisierten Austausch könnte die Weiterbildung wieder mehr in den Fokus des alltäglichen Bewusstseins rücken und langsam an Wichtigkeit gewinnen.

Das elektronische Logbuch (E-Logbuch) und die Weiterbildungsnovelle brechen die starre Schablone auf und lassen sie atmen, sodass wir sie hoffentlich an zukünftige Entwicklungen schneller anpassen können.

Das klingt naiv? Ist es auch. Aber es ist auch eine Chance.

\section{Anstellung \\ in Vertragsarztpraxis ohne Facharztqualifikation nicht möglich}

Das Landessozialgericht Bayern stellte mit Leitsatzurteil klar, dass angestellte Ärztinnen und Ärzte bei fehlender Weiterbildung nicht berechtigt sind, fachärztliche Leistungen $\mathrm{zu}$ Lasten der Kassenärztlichen Vereinigung zu erbringen. Der Versorgungsauftrag könne mit Angestellten ohne Facharztqualifikation nicht erfüllt werden. Auch Bestandsschutz könne dem nicht entgegengehalten werden. Die Folge - Die Anstellungsgenehmigung wurde aufgehoben. (Az. L12 KA 8/19)

Im vorliegenden Fall wehrte sich ein Vertragsarzt gegen die Aufhebung einer Anstellungsgenehmigung einer angestellten Ärztin. Die Anstellungsgenehmigung hatte bereits seit dem Jahre 1993 Bestand. Als Grund sicht des Vertragsarztes, sondern selbstständig in eigener Verantwortung durch. Die Behandlung durch angestellte Ärztinnen und Ärzte dürfe der Behandlung durch die Niedergelassenen selbst hinsichtlich der Qualität in Nichts nachstehen. Auch die Vorschriften zur vertragsärztlichen Bedarfsplanung seien nur dann sinnvoll zu praktizieren, wenn Praxisinhaber und Angestellte derselben Gebietsgruppe angehören würden.

Ein weiterer Grund, der nach Auffassung des Landessozialgerichts Bayern gegen die Anstellung gebietsfremder Ärztinnen und Ärzte spreche, sei, dass mit dem EBM2000plus zum zweiten Quartal 2005 eine strikte Trennung der haus- und fachärztlichen Versorgung vorgenommen worden sei. Durch das Sozialgesetzbuch sei damit vorgegeben worden, dass alle im EBM aufgeführten Leistungen entweder dem hausärztlichen oder dem fachärztlichen Bereich zuzuordnen seien. Die Leistungen im EBM untergliederten sich weiter anhand der Arztgruppen. Der EBM gestatte die Abrechnung von Leistungen nur denjenigen Ärztinnen und Ärzten, die aufgrund entsprechender Weiterbildung der jeweiligen Arztgruppe angehörten. Dies gelte gleichermaßen für die arztgruppenübergreifenden Leistungen, da diese aufgrund des Hinweises in der Präambel des Abschnitts II. des EBM als ergänzende Leistungen zu den arztgruppenspezifischen Leistungen abrechenbar seien. Genauso seien die arztgruppenübergreifenden speziellen Leistungen zu beurteilen, die als Abrechnungsvoraussetzung für Leistungen des Abschnitts IV. die Facharztanerkennung forderten.

Das Gericht sprach dem klagenden Vertragsarzt keinen Bestandsschutz zu. Die Aufhebung der Anstellungsgenehmigung für die Zukunft stelle keinen unverhältnismäßig schweren Eingriff in die Rechte der Betroffenen dar. $\mathrm{Zu}$ Gunsten des Gemeinwohls, insbesondere zur Sicherstellung einer qualitativ hochwertigen Versorgung der Versicherten, sei die Aufhebung der Anstellungsgenehmigung erforderlich. Der Verhältnismäßigkeitsgrundsatz sei gewahrt, da die angestellte Ärztin alternative Berufstätigkeiten wahrnehmen könne, der Vertragsarzt könne eine Ärztin oder einen Arzt mit entsprechender Qualifikation einstellen.

\section{Praxishinweis}

Für die Praxis hat das Urteil zur Folge, dass angestellte Ärztinnen und Ärzte ohne Facharztqualifikation in der vertragsärztlichen Versorgung mit einer Anstellungsgenehmigung weiter tätig sein können, solange die Genehmigung seitens der Kassenärztlichen Vereinigung nicht aufgehoben wird. Mit einer Aufhebung der noch bestehenden rechtswidrigen Anstellungsgenehmigungen muss gerechnet werden, da die Kassenärztliche Bundesvereinigung gegenüber den Landeskassenärztlichen Vereinigungen auf die Aufhebung drängt. Nicht betroffen von diesem Urteil sind selbstverständlich angestellte Ärztinnen und Ärzte in Weiterbildung zur Fachärztin bzw. zum Facharzt.

Bei Fragen zu diesen oder anderen berufsrechtlichen Angelegenheiten kontaktieren Sie gerne die für BDI-Mitglieder kostenfreie Rechtsberatung: rechtsberatung@bdi.de oder 0611-1813317.

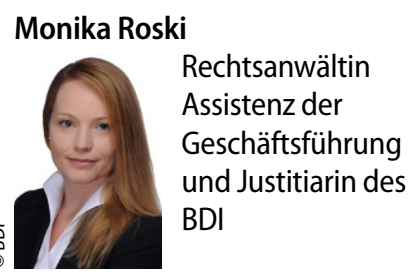




\section{Seminarwoche \\ Innere Medizin \\ kompakt \\ Pörtschach am Wörthersee (A) \\ 29.8.-3.9.2021
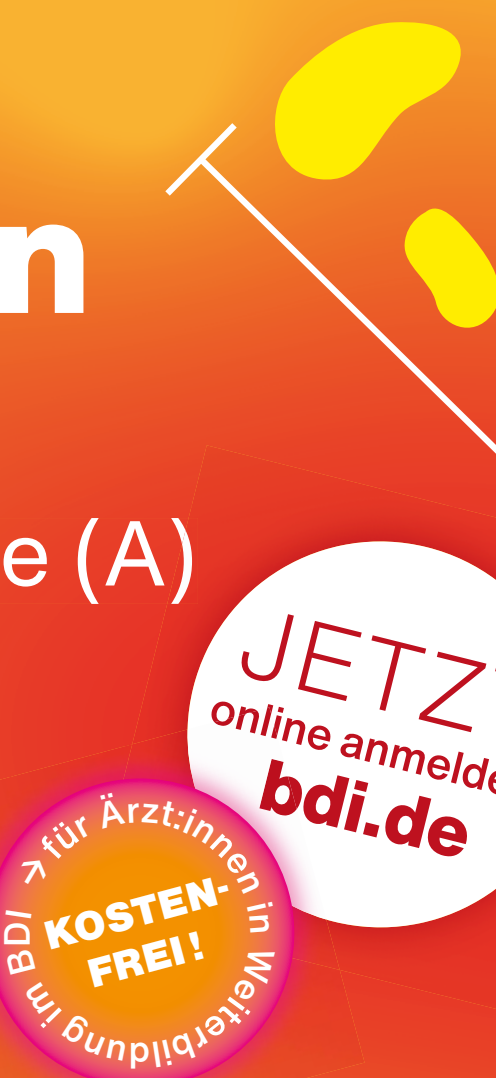

$\rightarrow$ Schwerpunkte: Gastroenterologie • Hepatologie • Pneumologie • Infektiologie • Kardiologie • Rheumatologie • Klinische Pharmakologie • Endokrinologie • Onkologie • Klinische Falldiskussionen • EKG Refresher • Ultraschall-Refresher Abdomen • Ultraschall-Refresher Gefäße

Wissenschaftliche Leitung: Prof. Dr. med. Bernhard Wörmann (Berlin) • Prof. Dr. med. Andreas Tromm (Hattingen) Zertifizierung: Diese Seminarwoche wird mit vorauss. 40 DFP-Punkten von der Österreichischen Ärztekammer approbiert. Die DMP Anerkennung wird beantragt.

\section{$\rightarrow$ Praktischer Kurs: Echokardiographie Grundkurs}

Kursleitung: Dr. med. Wolfgang Kirste (Schwabach) • Dr. med. univ. Mathias Ausserwinkler (Villach)

Zertifizierung: Dieser Kurs wird mit vorauss. 40 DFP-Punkten von der Österreichischen Ärztekammer approbiert.

Praktischer Kurs kostenpflichtig: $880 €$ regulär / $484 €$ für BDI-Mitglieder

Tagungsort: Congress Center Wörthersee • Hauptstraße 203 • 9210 Pörtschach am Wörthersee (A) 